\title{
Correction to: Sleep Quality in Autism from Adolescence to Old Age by Jovevska S, et al. Autism Adulthood 2020;2(2):152-162; DOI: 10.1089/aut.2019.0034
}

$\mathbf{I}^{\mathrm{N}}$

N THE JunE 2019 issue of Autism in Adulthood (vol. 2, no. 2; 152-162), there were errors in reporting some statistics in the article entitled Sleep Quality in Autism from Adolescence to Old Age by Jovevska S, Richdale AL, Lawson LP, Arnold SRC, and Trollor JN.

First, in the table of correlations (Table 5) on page 158, a significant correlation with sex was omitted. Sex was also significantly correlated with sleep onset latency in the ASD group $\mathrm{r}(282)=.156, p<.01$.

Original Table 5

Table 5. Correlations Between Sleep Variables AND AQ-Short, SeX, Demographic, Mental Health, and Medication

\begin{tabular}{|c|c|c|c|c|c|c|}
\hline \multirow[b]{2}{*}{ Variable } & \multicolumn{2}{|c|}{ Sleep quality } & \multicolumn{2}{|c|}{ SoL } & \multicolumn{2}{|c|}{ Total night sleep } \\
\hline & $\begin{array}{c}\text { Comparison } \\
\text { group }\end{array}$ & $\begin{array}{l}\text { Autistic } \\
\text { group }\end{array}$ & $\begin{array}{c}\text { Comparison } \\
\text { group }\end{array}$ & $\begin{array}{l}\text { Autistic } \\
\text { group }\end{array}$ & $\begin{array}{c}\text { Comparison } \\
\text { group }\end{array}$ & $\begin{array}{l}\text { Autistic } \\
\text { group }\end{array}$ \\
\hline AQ-short & $\begin{array}{c}0.268^{\mathrm{a}} \\
(n=211)\end{array}$ & $\begin{array}{c}0.245^{\mathrm{a}} \\
(n=269)\end{array}$ & - & - & $\begin{array}{l}-0.152^{\mathrm{b}} \\
(n=222)\end{array}$ & $\begin{array}{l}-0.154^{\mathrm{c}} \\
(n=281)\end{array}$ \\
\hline Sex & - & $\begin{array}{c}0.297^{\mathrm{a}} \\
(n=268)\end{array}$ & - & - & - & - \\
\hline Employed & $\begin{array}{c}0.189^{c} \\
(n=209)\end{array}$ & - & $\begin{array}{c}0.147^{\mathrm{b}} \\
(n=219)\end{array}$ & - & - & $\begin{array}{c}0.164^{\mathrm{c}} \\
(n=254)\end{array}$ \\
\hline $\begin{array}{l}\text { Mental } \\
\text { health } \\
\text { condition }\end{array}$ & $\begin{array}{l}-0.408^{\mathrm{a}} \\
(n=212)\end{array}$ & $\begin{array}{l}-0.310^{\mathrm{a}} \\
(n=272)\end{array}$ & $\begin{array}{l}-0.209^{\mathrm{c}} \\
(n=223)\end{array}$ & $\begin{array}{l}-0.195^{\mathrm{a}} \\
(n=287)\end{array}$ & $\begin{array}{c}0.136^{\mathrm{b}} \\
(n=222)\end{array}$ & - \\
\hline Medication & $\begin{array}{l}-0.229^{c} \\
(n=218)\end{array}$ & $\begin{array}{l}-0.292^{\mathrm{a}} \\
(n=276)\end{array}$ & - & $\begin{array}{l}-0.132^{\mathrm{b}} \\
(n=291)\end{array}$ & - & - \\
\hline
\end{tabular}

Only significant correlations are reported.

${ }^{\mathrm{a}} p \leq 0.001$.

${ }^{\mathrm{b}} p<0.05$.

${ }^{\mathrm{c}} p \leq 0.01$.
Corrected Table 5

Table 5. Correlations Between Sleep Variables AND AQ-Short, Sex, Demographic, Mental Health, and Medication

\begin{tabular}{|c|c|c|c|c|c|c|}
\hline \multirow[b]{2}{*}{ Variable } & \multicolumn{2}{|c|}{ Sleep quality } & \multicolumn{2}{|c|}{ SoL } & \multicolumn{2}{|c|}{ Total night sleep } \\
\hline & $\begin{array}{c}\text { Comparison } \\
\text { group }\end{array}$ & $\begin{array}{l}\text { Autistic } \\
\text { group }\end{array}$ & $\begin{array}{l}\text { Comparison } \\
\text { group }\end{array}$ & $\begin{array}{l}\text { Autistic } \\
\text { group }\end{array}$ & $\begin{array}{c}\text { Comparison } \\
\text { group }\end{array}$ & $\begin{array}{c}\text { Autistic } \\
\text { group }\end{array}$ \\
\hline AQ-short & $\begin{array}{c}\mathbf{0 . 2 6 0} \mathbf{a}^{\mathbf{a}} \\
(n=211)\end{array}$ & $\begin{array}{c}0.245^{\mathrm{a}} \\
(n=269)\end{array}$ & - & - & $\begin{array}{l}-0.152^{\mathrm{b}} \\
(n=222)\end{array}$ & $\begin{array}{l}-0.154^{\mathrm{c}} \\
(n=281)\end{array}$ \\
\hline Sex & - & $\begin{array}{c}0.297^{\mathrm{a}} \\
(n=268)\end{array}$ & - & $\begin{array}{c}0.156^{c} \\
(n=282)\end{array}$ & - & - \\
\hline Employed & $\begin{array}{c}0.189^{c} \\
(n=209)\end{array}$ & - & $\begin{array}{c}0.147^{\mathrm{b}} \\
(n=219)\end{array}$ & - & - & $\begin{array}{r}0.164^{\mathrm{c}} \\
(n=254)\end{array}$ \\
\hline $\begin{array}{l}\text { Mental } \\
\text { health } \\
\text { condition }\end{array}$ & $\begin{array}{l}-0.408^{\mathrm{a}} \\
(n=212)\end{array}$ & $\begin{array}{l}-0.310^{\mathrm{a}} \\
(n=272)\end{array}$ & $\begin{array}{l}-0.209^{c} \\
(n=223)\end{array}$ & $\begin{array}{l}-0.195^{\mathrm{a}} \\
(n=287)\end{array}$ & $\begin{array}{c}0.136^{\mathrm{b}} \\
(n=222)\end{array}$ & - \\
\hline Medication & $\begin{array}{l}-0.229^{c} \\
(n=218)\end{array}$ & $\begin{array}{l}-0.292^{\mathrm{a}} \\
(n=276)\end{array}$ & - & $\begin{array}{l}-0.132^{\mathrm{b}} \\
(n=291)\end{array}$ & - & - \\
\hline
\end{tabular}

Only significant correlations are reported.

${ }^{\mathrm{a}} p \leq 0.001$.

${ }_{p} p<0.05$.

${ }^{\mathrm{c}} p \leq 0.01$

Second, on page 157, correlations were reported between sex and mental health problems and sex and medication. This is incorrect as these are both dichotomous variables.

"In the autistic group, sex was also associated with AQ-short, $r(n=294)=0.179, p=0.002$; mental health condition, $r(n=187)=-0.394, p<0.001$; and medication, $r(n=187)=-0.266, p<0.001$."

The correct statistics are:

"In the autistic group, sex was also associated with AQ-short, $r(n=294)=0.179, p=0.002$; mental health condition, $\chi^{2}(1)=4.973, p=0.026, \phi=-0.146$ and medication, $\chi^{2}(1)=5.062, p=0.024, \phi=-0.146 . "$

The online version has been corrected to reflect this. The authors apologize for these errors, which did not affect the interpretations made. 